\title{
Valuation of Flexible Transmission Investment Options under Uncertainty
}

\author{
Ioannis Konstantelos, Member, IEEE, and Goran Strbac, Member, IEEE
}

\begin{abstract}
Significant uncertainty surrounds the future development of electricity systems, primarily in terms of size, location and type of new renewable generation to be connected. In this paper we assess the potential for flexible network technologies, such as phase-shifting transformers, and nonnetwork solutions, such as energy storage and demand-side management, to constitute valuable interim measures within a long-term planning strategy. The benefit of such flexible assets lies not only in the transmission services provided but also in the way they can facilitate and de-risk subsequent decisions by deferring commitment to capital-intensive projects until more information on generation development becomes available. A novel stochastic formulation for transmission expansion planning is presented that includes consideration of investment in these flexible solutions. The proposed framework is demonstrated with a case study on the IEEE-RTS where flexible technologies are shown to constitute valuable investment options when facing uncertainties in future renewable generation development.
\end{abstract}

Index Terms-Benders decomposition, energy storage, stochastic programming, transmission expansion planning.

\section{NOMENCLATURE}

$\mathrm{T}^{\mathrm{H}}$ HE mathematical symbols in this paper are as follows.

\section{A. Sets and indices}

$\Omega_{S}$

$\Omega_{M}$

$\Omega_{E}$

$\Omega_{B}$

$\Omega_{T}^{b}$

$\Omega_{N}$

$\Omega_{G}$

$\Omega_{L}$

$\Omega_{W}$

$\sigma_{b}$

$\varepsilon_{m}$

$\Phi_{m}^{0}$

$\Phi_{m}^{\gamma}$
Set of scenarios.

Set of scenario tree nodes, indexed $m$.

Set of epochs, indexed $e$.

Set of demand blocks, indexed $b$.

Set of periods in demand block $b$, indexed $t$.

Set of system buses, indexed $n$.

Set of generation units, indexed $g$.

Set of transmission lines, indexed $l$.

Set of reinforcement options, indexed $w$.

The first period of demand block $b$.

The epoch to which node $m$ belongs.

A time-ordered set that contains all parents of node $m$, including $m$ as the last element.

A time-ordered set that contains all parents of node $m$, from the first stage up to stage $\varepsilon_{m}-\gamma$, where $\gamma$ is integer.

\section{B. Input parameters}

$\bar{p}_{m, g}$

Maximum stable generation for unit $g$ under scenario node $m$ (MW).

$\kappa_{g}^{G}$ Operating cost of generating unit $g(£ / \mathrm{MWh})$.

I. Konstantelos and G. Strbac are with the Control and Power Group, Faculty of Electrical and Electronic Engineering, Imperial College London, UK.
$D_{t, n}$

$F_{l}^{0}$

$b_{l}$

$u_{l}$

$v_{l}$

$\chi_{l}$

$I_{n, l}$

$J_{n, g}$

$\pi_{m}$

$\tau_{t}$

$\bar{F}_{w}$

$\bar{\xi}$

\section{Decision variables}

$B_{m, l, w}$

$\tilde{F}_{m, l}$

$Q_{m, l}$

$\tilde{Q}_{m, l}$

$H_{m, n}$

$\widetilde{H}_{m, n}$

$p_{m, t, g}$ $f_{m, t, l}$ $(\mathfrak{f} / \mathrm{yr})$. line $l$. node $m$. $m$.
Demand at bus $n$ in period $t$ (MW).

Initial capacity of transmission line $l(\mathrm{MW})$. Susceptance of transmission line $l$ (p.u.).

Start node for line $l$.

Destination node for line $l$.

Length of line $l(\mathrm{~km})$.

Bus-to-line incidence matrix.

Bus-to-generation incidence matrix.

Probability of scenario node $m$.

Time duration of period $t$ (hours).

Maximum capacity provided by expansion option $w$ (MW).

Maximum angle of phase-shifter.

Maximum charge/discharge rate of storage device (MW).

Energy capacity of storage device (MWh).

Annual capital cost of reinforcement option $w$ (£/km-yr).

Annual capital cost of one phase-shifter (£/yr).

Annual capital cost of one storage device

Build time of reinforcement option $w$.

Build time of a phase-shifting transformer.

Build time of a storage device.

Cumulative discount factor for investment cost in epoch $e$.

Cumulative discount factor for operation cost in epoch $e$.

System balance penalty constant (£/MWh).

Binary variable signifying the choice of reinforcement option $w$ in scenario node $m$ for

State variable of aggregate extra capacity available to line $l$ in scenario node $m$.

Binary variable signifying installation of phase-shifter on line $l$ in scenario node $m$.

Aggregate binary state variable denoting presence of phase-shifter on line $l$ in scenario

Binary variable signifying installation of storage device at bus $n$ in scenario node $m$.

Aggregate binary state variable denoting presence of storage at bus $n$ in scenario node

Output of unit $g$ at operating point $(m, t)$.

Power flow on line $l$ at operating point $(m, t)$. 


\begin{tabular}{|c|c|}
\hline$\theta_{m, t, n}$ & Bus angle of $n$ at operating point $(m, t)$. \\
\hline$\xi_{m, t, l}$ & $\begin{array}{l}\text { Angle of phase-shifter at line } l \text { at operating } \\
\text { point }(m, t) \text {. }\end{array}$ \\
\hline$h_{m, t, n}$ & $\begin{array}{l}\text { Output of storage device at bus } n \text { at operating } \\
\text { point }(m, t) \text {. }\end{array}$ \\
\hline$\tilde{h}_{m, t, n}$ & $\begin{array}{l}\text { State of charge of storage device at bus } n \text { at } \\
\text { operating point }(m, t) \text {. }\end{array}$ \\
\hline$d_{m, t, n}$ & $\begin{array}{l}\text { Curtailed demand at bus } n \text { at operating point } \\
(m, t) \text {. }\end{array}$ \\
\hline
\end{tabular}

\section{INTRODUCTION}

Many countries across the world have committed to a decarbonisation of their electricity system with legally binding targets. For example, the European Council has adopted a bill that dictates $20 \%$ reduction in $\mathrm{CO}_{2}$ emissions and $20 \%$ uptake of renewables in total energy consumption by 2020 [1]. Under this global drive towards renewable energy sources, the transmission infrastructure becomes one of the pillars of efficient energy markets and a key enabler for meeting new public policy goals. Given the unprecedented level of transmission investment needed for a transition towards a lowcarbon economy, it is essential to ensure that planning decisions are supported by a framework that encourages longterm economic efficiency and considers the new challenges and opportunities that arise within modern energy systems.

Historically, network planning has involved little uncertainty regarding future plant developments. However, the rapid growth of renewable energy sources entails significant uncertainty regarding the type and size of new generators, rendering the network planner unable to make fully-informed commitments to long-term projects. In addition, renewable generators are typically located further away from load centers and have shorter construction times than conventional plants. In many jurisdictions, this situation is further aggravated by the growing public opposition towards establishing new power corridors; new entrants may be connected to the system faster than the main grid can be upgraded to accommodate them, while planners are entrenched in time-consuming processes to secure planning permissions [2]. The result of keeping a reactive stance is constrained access to merit plants, mounting congestion costs and reduced utilization of low-carbon energy sources.

Under this new paradigm, network planners must depart from traditional planning practices and adopt an anticipatory framework, where expansion projects are undertaken beyond the immediate system needs. In response to this new reality, recent advances in planning models have moved from deterministic to stochastic approaches, where optimal decisions are taken on the basis of future adaptability against a set of envisaged scenarios. However, the irreversible nature of such capital-intensive investments gives rise to a material risk of stranded assets or premature lock-in to sub-optimal investment paths in the case of unfavorable scenario realizations. Long build times of transmission assets further exacerbate these risks, severely limiting the viability of adopting a 'wait-and-see' approach.

However, along with the challenges that arise within contemporary system planning, new opportunities are enabled by novel technologies. Although conventional capacity reinforcements are the backbone for enabling large power transfer between areas, investment in non-network technologies such as storage and demand-side management (DSM) can form a viable strategy for alleviating congestion. DSM is capable of rescheduling energy consumption of flexible loads, while storage devices enable arbitrage between periods of low and high energy scarcity and congestion, both enhancing the ability of the system to accommodate intermittent renewables [3]. In a similar manner, phaseshifting transformers can alleviate congestion by controlling flow of power over the network [4].

One chief aspect of the afore-mentioned technologies is that they enable higher utilization of the existing network infrastructure by providing flexibility in managing the demand-supply balance. Furthermore, they can be instrumental in preserving system security through provision of post-fault corrective actions [5]. This results not only in more economic operation than when relying solely on preventive generation dispatch, but also in a reduced need for transmission investment, which is traditionally driven by security considerations.

Beyond the afore-mentioned benefits of investment and operation cost savings, there is a less-documented aspect of interest. When examined in a stochastic setting, one farreaching advantage of incorporating such flexible assets in the planning process is the possibility to defer commitment to major conventional projects until the need for such investment is fully established. Investment options that are not part of the optimal investment plan due to the availability of more efficient long-term alternatives (such as establishing a new power corridor), may become integral parts of a long-term investment strategy when the planner is facing uncertainty. In other words, interim measures can be useful in 'buying time' until more information regarding the generation system evolution is available, thus rendering viable a 'wait-and-see' strategy that would otherwise be too costly.

This paper aims at demonstrating that investment in nonconventional assets can hold significant value due to the ability to keep future options open and defer commitments to costly reinforcements. In addition, we show that deterministic planning approaches can systematically undervalue the benefit that such flexible assets can provide. To demonstrate the above concepts we propose a novel model for multi-stage stochastic transmission planning. We assume that a central planner responsible for maximizing expected social welfare is facing exogenous uncertainty on future renewable generation connections. The model identifies the optimal investment policy across multiple asset types while abiding to the N-1 security criterion. The contributions of this paper are threefold:

- A comprehensive stochastic planning framework is proposed for evaluating different investment options under uncertainty.

- An integrated algorithm that addresses the model's increased computational complexity is developed. 
- The presented case studies provide insight in the role that flexible and non-conventional assets can play in accommodating renewables and their option value is quantified.

The remaining sections of this paper are organized as follows: Section II explores existing approaches for modelling transmission planning under uncertainty and highlights the contributions of the present research. Section III presents the problem formulation and proposed solution approach. Section IV showcases numerical results obtained by case studies on the IEEE-RTS. Section V provides recommendations for future work and concludes.

\section{LITERATURE REVIEW}

Transmission planning under uncertainty is an active research area that has received considerable attention in the past. However, most existing formulations (e.g. [6], [7]) are limited to a static description of uncertainty, employing probabilistic models. Such frameworks are limiting due to the inherent inability to consider openings for strategic action. Strategic opportunities arise in all dynamic decision systems under uncertainty and are due to the inter-temporal resolution of uncertainty [8]. It follows that a multi-stage problem formulation is required to shift from 'now-or-never' decisions to an integrated strategy that considers the value of delaying decisions until more information is known. The importance of managerial flexibility in capital budgeting decisions is recognized by many researchers [9] and has been crystallized in a valuation framework known as Real Options Analysis (ROA), with several applications on transmission planning (e.g. [10]). Although Real Options Analysis can take many forms, from binomial trees to Monte Carlo simulation, its application scope is usually limited to a small set of candidate options. Tackling larger systems requires a shift from valuation techniques to a systematic optimization framework. The tight links between option theory and stochastic programming have been highlighted [11]; it is the appropriate framework for modeling the path-dependency problem that characterizes real option valuation. In this vein, some recent studies have employed multi-stage stochastic programming to model transmission investment under uncertainty, as in [12] and [13]. However, in all existing formulations, uncertainty is modeled via a scenario fan that may limit the resolution of uncertainty to only the first state transition. As discussed in [14], this approach simplifies the underlying uncertainty structure and is not suitable for describing a stage-wise decision process. In this paper we enhance existing approaches by providing a generic stochastic problem formulation for arbitrary scenario tree structures with multiple nodes and stages, in line with proposals in [15] and [16]. Note that our model focuses solely on exogenous uncertainty and ignores the potential interactions between transmission and generation strategic investment decisions, although these interactions exist and may have a significant impact on investment as shown in [17] and [18]. However, full consideration of such interactions makes the model too complex and can lead to intractability, rendering problematic the long-term strategic analysis of even simple systems.

\section{MATHEMATICAL FORMULATION}

We formulate the stochastic planning problem as a mixed integer-linear problem (MILP). Uncertainty is modelled in the form of a multi-stage scenario tree of $\left|\Omega_{M}\right|$ nodes spanning $\left|\Omega_{E}\right|$ epochs (equivalent to stages) and portrays the possible states and transition probabilities. For the purposes of this research we adopt the node-variable approach, where investment and operation variables are defined in terms of decision points, foregoing the introduction of nonanticipativity constraints [19]. In general, a scenario tree provides a coherent description of the future evolution of one or more system parameters. It is usually the product of expert opinion, industry surveys and analysis of the underlying market dynamics. In the presented model, the scenario tree describes the evolution of generation capacity and is incorporated in the model by introducing the node-variable input parameters $\bar{p}_{m, g}$. It follows that other sources of uncertainty can be included in a similar manner. For example, a bivariate scenario tree that describes future evolution of phase-shifter and storage device investment costs can be constructed instead and incorporated in the model by rendering the corresponding parameters $\kappa^{Q}$ and $\kappa^{H}$ to vary across different scenario nodes, as in $\kappa_{m}^{Q}$ and $\kappa_{m}^{H}$. Naturally, scenario trees grow exponentially with the number of variables they describe and thus appropriate reduction or sampling techniques should be applied (e.g.[14]).

\section{A. Computational Challenges}

One of the key challenges of stochastic problem formulations is problem size; the number of operational and investment variables and the associated constraints grows rapidly with the number of scenarios and stages. One way to address this issue is to use a decomposition technique. The application of Benders decomposition [20] in the context of power systems has been carried out successfully in the past [21]. Transmission and generation planning models in particular exhibit problem structures highly exploitable by such decomposition methods [22]. Benders is an iterative method that decomposes the original problem into a master problem (transmission investment) and several sub-problems (power system operation). The objective function of the master problem is derived by substituting the operating cost component of the original problem with an estimate. At each iteration $k$, the master problem determines all investment decisions. In turn, all the operational sub-problems are solved subject to these decisions. The Lagrange multipliers of the sub-problems' complicating constraints are then used to build the Benders cuts which are appended to the master problem. In the classical Benders approach, one extra constraint is generated per iteration. Here, we have employed a multicut Benders decomposition formulation [23], where a set of $\left|\Omega_{M}\right|\left|\Omega_{B}\right|$ cuts is produced per iteration, improving representation of operational costs in the master problem and leading to faster convergence at the expense of additional constraints. 
To maintain a tractable operation problem size, we assume that each year consists of several representative demand blocks; time-coupling is considered within periods of the same block but ignored in-between blocks. This is a reasonable assumption in the case of storage devices, which are typically scheduled on an intra-week basis.

Another major source of computational complexity is consideration of N-1 security criteria. The traditional securityconstrained optimal power flow (SCOPF) involves exhaustive modelling of $\mathrm{N}-1$ security constraints; this entails the introduction of extra variables and constraints to represent post-fault system operation. To address this issue, several different methods have been proposed in the literature (see [24] for a comprehensive overview). The basic idea is that only a few contingencies are binding and require preventive and/or corrective re-dispatch; post-fault constraints related to non-binding contingencies are redundant and can be ignored (e.g. [25], [26]). However, all existing methods consider operation problems in isolation, not dealing with investment decisions that impose a more limiting coupling structure. In this research we propose an iterative contingency screening approach that is efficiently combined with the investmentoperation Benders decomposition scheme. The detailed mathematical formulation follows.

\section{B. Investment Master Problem}

The master problem is independent of non-complicating variables and approximates the subproblem value using the scalar decision variables $\alpha_{m, b}$. As Benders cuts are being added to the master problem, the subproblem is built up from below until the master problem equals the original problem.

where

$$
\underset{F, Q, H}{\min }\left\{\sum_{\forall m} \pi_{m} r_{\varepsilon_{m}}^{I} \psi_{m}+\sum_{\forall m, b} \pi_{m} \alpha_{m, b}\right\}
$$

$$
\psi_{m}=\sum_{\forall l}\left(B_{m, l, w} \kappa_{w}^{F} \chi_{l}+Q_{m, l} \kappa^{Q}\right)+\sum_{\forall n} H_{m, n} \kappa^{H}
$$

Subject to

$$
\begin{gathered}
\tilde{F}_{m, l}=\sum_{w \in \Omega_{W}} \sum_{\phi \in \Phi_{m}^{\gamma_{w}^{F}}} B_{\phi, l, w} \bar{F}_{w}, \forall m \in \Omega_{M}, l \in \Omega_{L} \\
\tilde{Q}_{m, l}=\sum_{\phi \in \Phi_{m}^{\gamma^{Q}}} Q_{\phi, l}, \forall m \in \Omega_{M}, l \in \Omega_{L} \\
\widetilde{H}_{m, n}=\sum_{\phi \in \Phi_{m}^{\gamma^{H}}} H_{\phi, n}, \forall m \in \Omega_{M}, n \in \Omega_{N} \\
B_{m, l} \in\{0,1\}, \quad \forall m \in \Omega_{M}, l \in \Omega_{L} \\
Q_{m, l} \in\{0,1\}, \quad \forall m \in \Omega_{M}, l \in \Omega_{L} \\
H_{m, n} \in\{0,1\}, \quad \forall m \in \Omega_{M}, n \in \Omega_{N} \\
\alpha_{m, b} \geq \omega_{m, b}^{k-1}+\sum_{\forall l} \lambda_{m, b, l}^{F, k-1}\left(\tilde{F}_{m, l}-\widetilde{F}_{m, l}^{k-1}\right) \\
+\sum_{\forall l}^{\forall} \lambda_{m, b, l}^{Q, k-1}\left(\widetilde{Q}_{m, l}-\widetilde{Q}_{m, l}^{k-1}\right) \\
+\sum_{\forall n} \lambda_{m, b, n}^{H, k-1}\left(\widetilde{H}_{m, n}-\widetilde{H}_{m, n}^{k-1}\right) \\
, \forall m \in \Omega_{M}, \forall b \in \Omega_{B}
\end{gathered}
$$

Equation (1a) is the master problem objective function that corresponds to the expected discounted system cost over the study horizon. Our model assumes that system operations take place in a perfectly competitive power market. The objective function consists of probability-weighted investment costs related to line reinforcements, phase-shifters and storage devices as well as an approximation of the expected operating cost, progressively informed through the appended Benders cuts. Constraint (1c) states that the amount of transmission capacity available at node $m$ is the aggregated capacity constructed over all previous epochs of the corresponding scenario path, while considering commissioning delays due to build time. Constraints (1d)-(1e) impose a similar relation between control and state variables for investment in phaseshifters and storage. The binary nature of investment decision variables is stated in constrains (1f)-(1h). Constraints (1i) are the Benders cuts appended to the master problem at the $k^{\text {th }}$ iteration. Each cut provides a lower bound estimate for the operating cost of block $(m, b)$. It is formulated in terms of the trial investment solutions of the previous iteration $(k-1)$, the optimal objective value of each operation subproblem $\omega_{m, b}^{k-1}$ and the Lagrange multipliers of subproblem constraints that couple investment decisions to operation.

\section{Operation Subproblem}

At each iteration $k$, the subproblem utilizes the master's trial solution (i.e. investment state variables $\widetilde{F}^{k}, \widetilde{Q}^{k}, \widetilde{H}^{k}$ ) in the constraints that couple investment with operation. Note that operation subproblems can be run in parallel.

$$
\omega_{m, b}^{k}=\min _{p, d}\left\{r_{\varepsilon_{m}}^{O} \sum_{t \in \Omega_{T}^{b}} \tau_{t}\left[\sum_{\forall g} p_{m, t, g} \kappa_{g}^{G}+\sum_{\forall n} d_{m, t, n} \Gamma\right]\right\}
$$

Subject to

$$
\begin{gathered}
F_{m, l}^{*}=\widetilde{F}_{m, l}^{k}: \lambda_{m, b, l}^{F, k}, \forall l \in \Omega_{L} \\
Q_{m, l}^{*}=\widetilde{Q}_{m, l}^{k}: \lambda_{m, b, l}^{Q, k}, \forall l \in \Omega_{L} \\
H_{m, n}^{*}=\widetilde{H}_{m, n}^{k}: \lambda_{m, b, n}^{H, k}, \forall n \in \Omega_{N} \\
\sum_{\forall g} J_{n, g} p_{m, t, g}+\sum_{\forall l} I_{n, l} f_{m, t, l}=, \forall t \in \Omega_{T}^{b} \\
D_{t, n}-d_{m, t, n}+h_{m, t, n} \quad, \forall n \text { in } \Omega_{N} \\
0 \leq p_{m, t, g} \leq \bar{p}_{m, g}, \forall t \in \Omega_{T}^{b}, \forall g \in \Omega_{G} \\
f_{m, t, l}=b_{l}\left(\theta_{m, t, u_{l}}-\theta_{m, t, v l}+\xi_{m, t, l}\right), \forall t \in \Omega_{T}^{b} \\
\left|f_{m, t, l}\right| \leq F_{l}^{0}+F_{m, l}^{*}, \forall t \in \Omega_{T}^{b}, \forall l \in \Omega_{L} \\
\left|\xi_{m, t, l}\right| \leq Q_{m, n}^{*} \bar{\xi}, \forall t \in \Omega_{T}^{b}, \forall l \in \Omega_{L} \\
\left|h_{m, t, n}\right| \leq H_{m, n}^{*} \bar{h}, \forall t \in \Omega_{T}^{b}, \forall n \in \Omega_{N} \\
\widetilde{h}_{m, t, n} \leq \bar{\eta}, \forall t \in \Omega_{T}^{b}, \forall n \in \Omega_{N} \\
\tilde{h}_{m, t, n}=\widetilde{h}_{m, t-1, n}+h_{m, t, n} \tau_{t}, \forall t \in \Omega_{T}^{b} \backslash\left\{\sigma_{b}\right\}, \forall n \in \Omega_{N}
\end{gathered}
$$

$$
\begin{aligned}
& \forall c \in \Omega_{L}: \widetilde{\Delta}_{m, t, c}=1 \\
& \sum_{\forall g} J_{n, g} p_{m, t, g}+\sum_{\forall l} I_{n, l} f_{m, t, c, l}^{C}=\forall t \in \Omega_{T}^{b} \\
& D_{t, n}-d_{m, t, n}+h_{m, t, c, n}^{C} \quad, \forall n \in \Omega_{N} \\
& f_{m, t, c, l}^{C}=\left\{\begin{array}{cl}
b_{l}\left(\theta_{m, t, c, u_{l}}^{C}-\theta_{m, t, c, v_{l}}^{C}\right), & \forall t \in \Omega_{T}^{b} \\
0 & \forall l \in \Omega_{L}
\end{array}\right. \\
& \left|f_{m, t, c, l}^{C}\right| \leq\left\{\begin{array}{c}
F_{l}^{0}+\tilde{F}_{m, l}^{*} \text { if } c \neq l \quad \forall t \in \Omega_{T}^{b} \\
0 \text { if } c=l \quad, \forall l \in \Omega_{L}
\end{array}\right.
\end{aligned}
$$




$$
\begin{gathered}
\left|\xi_{m, t, c, l}^{C}\right| \leq\left\{\begin{array}{c}
Q_{m, n}^{*} \bar{\xi} \text { if } c \neq l \quad \forall t \in \Omega_{T}^{b} \\
0 \text { if } c=l, \forall l \in \Omega_{L}
\end{array}\right. \\
\left|h_{m, t, c, n}^{C}\right| \leq H_{m, l}^{*} \bar{h}, \forall t \in \Omega_{T}^{b}, \forall n \in \Omega_{N} \\
h_{m, t, c, n}^{C} \leq H_{m, n}^{*} \bar{\eta}-h_{m, t, n}, \forall t \in \Omega_{T}^{b}, \forall n \in \Omega_{N}
\end{gathered}
$$

The objective function (2a) is the sum of generation costs and penalized curtailed demand. The latter term communicates the need for investment to the master problem when the trial decisions cannot enforce system balance under all intact and N-1 conditions. Constraints (2b)-(2d) force the subproblem auxiliary investment decision variables $F^{*}, Q^{*}$ and $H^{*}$ to be equal to the optimal decisions supplied by the master problem at iteration $k$; an approach similar to the one adopted in [27]. The resulting Lagrange multipliers $\lambda^{F}, \lambda^{Q}$ and $\lambda^{H}$ are used to construct the Benders cuts to be appended in the next iteration.

Constraints (2e)-(2l) refer to pre-fault system operation. The system balance equation (2e) states that at each bus, the local generation and net of incoming/outgoing flows satisfy demand while considering operation of storage devices. The demand curtailment variable $d$ acts as a slack variable to avoid problem infeasibility and is penalized in the objective function according to the large positive constant $\Gamma$. Constraint (2f) provides limits on generation dispatch; time-variable limits should be introduced in the case of intermittent plants such as wind. Equation $(2 \mathrm{~g})$ defines how power is distributed over the network according to the dc formulation, while taking into account the effect of phase-shifters. Note that in the presented model we consider investments only in existing lines and ignore the effect of reinforcements on line reactance. If alternative corridors are to be considered, suitable disjunctive constraints should be included to enforce Kirchhoff's Voltage Laws on the candidate lines. Constraints $(2 \mathrm{~h})-(2 \mathrm{j})$ are the complicating constraints between investment and operation, ensuring that operation decision variables are limited by the investments commissioned at the corresponding scenario tree node. More precisely, constraint ( $2 \mathrm{~h}$ ) bounds line flows according to the existing capacity and trial solution of the master problem. Constraint (2i) allows operation of a phaseshifter only in the case that the phase-shifter is part of the trial investment solution (i.e. $Q_{m, n}^{*}=1$ ). In a similar fashion, constraint (2j) defines charge/discharge limits of each storage device as dependent on the corresponding investment decisions. Constraint (2k) limits the total energy stored according to the device's energy capacity $\bar{\eta}$. Equation (2l) states that the state of charge of a storage device is the summation of its previous state and the energy being charged/discharged in the current period. For simplicity reasons, our model assumes full efficiency in charging and discharging the storage units. Note that an initialization equation explicitly defining the state of charge at the first period of each block must be included in the formulation. Constraints similar to $(2 \mathrm{j}-2 \mathrm{l})$ can be used to model operation of DSM devices.

Constraints $(2 \mathrm{~m})-(2 \mathrm{r})$ refer to post-fault system operation. Note that post-fault constraints are included only for post-fault operating points found in the list of binding contingencies $\widetilde{\Delta}$. To model the corrective capabilities of phase-shifters and storage devices, post-fault operation variables are introduced; they are denoted by the superscript $C$ and an extra index $c \in \Omega_{L}$, that corresponds to line $c$ being in outage. Equation (2m) describes system balance after a line outage by coupling preventive generation dispatch with post-fault line flows and any available corrective actions. Constraints (2n)-(2q) are similar to their pre-fault counterparts $(2 \mathrm{~g})-(2 \mathrm{j})$, while also explicitly stating that by definition, when $c=l$, flow and phase-shift over the faulty line is zero. In addition, constraint (2r) ensures that the post-fault actions of storage abide to energy availability at the time.

\section{Benders Convergence Criterion}

The Benders convergence criterion (3a) is defined in terms of the difference between upper and lower problem bounds.

$$
\begin{gathered}
\frac{Z_{\text {upper }}^{k}-Z_{\text {lower }}^{k} \leq \bar{\zeta}}{Z_{\text {upper }}^{k}} \leq \pi_{m}^{I} r_{\varepsilon(m)} \psi_{m}^{k}+\sum_{\forall m, b} \pi_{m} \alpha_{m, b} \\
Z_{\text {upper }}^{k}=\sum_{\forall m} \pi_{\text {lower }}= \\
\sum_{\forall m}^{k} \pi_{m} r_{\varepsilon(m)}^{I} \psi_{m}^{k}+\sum_{\forall m, b}^{k} \pi_{m} \omega_{m, b}^{k}
\end{gathered}
$$

The threshold value $\bar{\zeta}$ should be a value close to 0 , in order to ensure close matching between the bounds of the problem.

\section{E. Contingency Screening Module}

Once convergence is achieved, the optimal investment and operation decisions should allow for feasible post-fault operation across all credible contingencies; each post-fault operating point $(m, t, c)$ is simulated and classified as binding or non-binding. This can be determined by evaluating the violation of the post-fault system balance equation while using the master problem's investment solution $\left\{\widetilde{F^{k}}, \widetilde{Q^{k}}, \widetilde{H}^{k}\right\}$ and subproblem's pre-fault operation solution $\{p, f, \xi, \bar{h}\}$. An optimization problem for each post-fault operating point $(m, t, c)$ is formulated as follows.

$$
\delta_{m, t, c}=\min _{d^{+}, d^{-}}\left\{\sum_{\forall n}\left(d_{m, t, c, n}^{+}+d_{m, t, c, n}^{-}\right)\right\}
$$

Subject to constraints (2m)-(2r). The post-fault system balance equation $(2 \mathrm{~m})$ is modified to incorporate two infeasibility slack variables; $d^{+}$and $d^{-}$signifying violations in the negative and positive direction respectively.

$$
\begin{aligned}
& \sum_{\forall g} J_{n, g} p_{m, t, g}+\sum_{\forall l} I_{n, l} f_{m, t, c, l}^{C}=D_{t, n}+{ }_{, n \forall \in \Omega_{N}} \\
& d_{m, t, c, n}^{+}-d_{m, t, c, n}^{-}+h_{m, t, c, n}^{C}
\end{aligned}
$$

Classification is achieved through quantification of the two slack variables and comparison with a threshold value $\bar{\delta}$, appropriately set close to 0 , as shown below.

$$
\Delta_{m, t, c}= \begin{cases}1 & \text { if } \delta_{m, t, c} \geq \bar{\delta} \\ 0 & \text { otherwise }\end{cases}
$$

In the case that $\sum_{\forall(m, t, c)} \Delta_{m, t, c}>0$, meaning that there are some post-fault violations, the problem must be solved again 
with an expanded list of binding-contingencies. To this end, equation (4d) is applied.

$$
\widetilde{\Delta}_{m, t, c}=\max \left(\widetilde{\Delta}_{m, t, c}, \Delta_{m, t, c}\right)
$$

Note that according to the expression above, if a post-fault operating point is clear under the current solution $\left(\Delta_{m, t, c}=0\right)$ but has been found to be binding in the past, its status is reinstated as binding. This ensures that any potentially-binding contingencies are always considered explicitly and the algorithm does not oscillate between solutions.

\section{F. Solution Algorithm}

For the sake of clarity, the complete solution algorithm is presented below.

\begin{tabular}{|c|c|}
\hline \multicolumn{2}{|c|}{ Algorithm 1 Benders with Decomposed SCOPF } \\
\hline Step 1. & $\widetilde{\Delta}_{m, t, c}=0, \forall(m, t, c)$ \\
\hline Step 2. & $\begin{array}{l}\text { Set Benders iteration index } k=1 \text { and discard al } \\
\text { appended Benders cuts from the master problem. }\end{array}$ \\
\hline Step 3. & $\begin{array}{l}\text { Solve the master problem subject to all appended } \\
\text { Benders cuts. }\end{array}$ \\
\hline Step 4. & $\begin{array}{l}\text { Solve all operation subproblems utilizing master } \\
\text { problem's trial investment decisions. }\end{array}$ \\
\hline Step 5. & $\begin{array}{l}\text { Check the convergence criterion (3a). If false, } \\
\text { construct and append all relevant Benders cuts, } \\
\text { update index } k=k+1 \text { and go to Step } 3 \text {. }\end{array}$ \\
\hline Step 6. & $\begin{array}{l}\text { Screen all post-fault operating points }(m, t, c) \\
\text { subject to the optimal investment and pre-faul } \\
\text { operating decisions and determine binding status } \\
\text { of each point according to }(4 \mathrm{c}) \text {. }\end{array}$ \\
\hline Step 7. & $\begin{array}{l}\text { If } \sum_{\forall(m, t, c)} \Delta_{m, t, c}=0 \text {, go to Step 8. Otherwise } \\
\text { update the } \widetilde{\Delta} \text { vector according to (4d) and go to } \\
\text { Step } 2 \text {. }\end{array}$ \\
\hline Step 8. & END \\
\hline
\end{tabular}

\section{IEEE-RTS CASE STUDY}

In this section we present a case study to showcase how the proposed stochastic framework can evaluate the benefit of different expansion options. By comparing system costs when allowing investment solely in conventional network reinforcements and when considering alternative technologies, we can investigate how the availability of flexible assets can impact transmission planning under uncertainty.

\section{A. Description}

The studies of this paper have been conducted using the IEEE 24-bus reliability system (IEEE-RTS) [28] that consists of 24 buses, 38 lines and 32 generation units. The topology has been preserved the same as in the original paper, with minimum stable generation for all units set to $0 \mathrm{MW}$. For the sake of model simplification, hydro units installed at bus 22 have been removed and all line lengths have been set to $50 \mathrm{~km}$. In addition, all lines have been initialized with just enough capacity to allow N-1 secure operation with no congestion in the first epoch. To avoid islanding, an extra line connecting buses 7-8 has been added. The operating cost of the three generation technologies, nuclear, coal and oil, have been set to
6,50 and 150£/MWh respectively. Total conventional generation capacity in the first epoch is $3,105 \mathrm{MW}$ while system peak load is assumed to stay unchanged at $2,850 \mathrm{MW}$.

The study horizon comprises of 3 five-year epochs over which an uncertain amount of wind generation is connected to bus 24. It is assumed that following suitable consultation and analysis, a scenario tree describing the possible future capacity evolution has been constructed, shown in Fig. 1. S1 represents full deployment of $1600 \mathrm{MW}$ of wind capacity, while no wind capacity is built under S4. The other two scenarios result in $800 \mathrm{MW}$ but differ in terms of time of deployment. Note that the presented case study focuses solely on the uncertainty regarding future penetration of renewables and thus all other parameters are considered to not vary across scenario nodes. Additional uncertainty sources can be incorporated in a straightforward manner, as outlined in section III.

To capture the different operating conditions, five demand blocks (168 hours each - equivalent to a week) are used; one for each calendar season and an extra block capturing peak demand conditions in late December. Each block is assumed to repeat 12.5 times (peak week occurs only once), with the corresponding sub-problems weighted accordingly. To ensure realistic inter-temporal behavior and dependence structure, the weekly wind and loading time series were extracted from measurements of Great Britain's aggregate wind production and electricity demand in 2012. Annual mean wind and demand factors are $32.5 \%$ and $65.8 \%$ respectively.

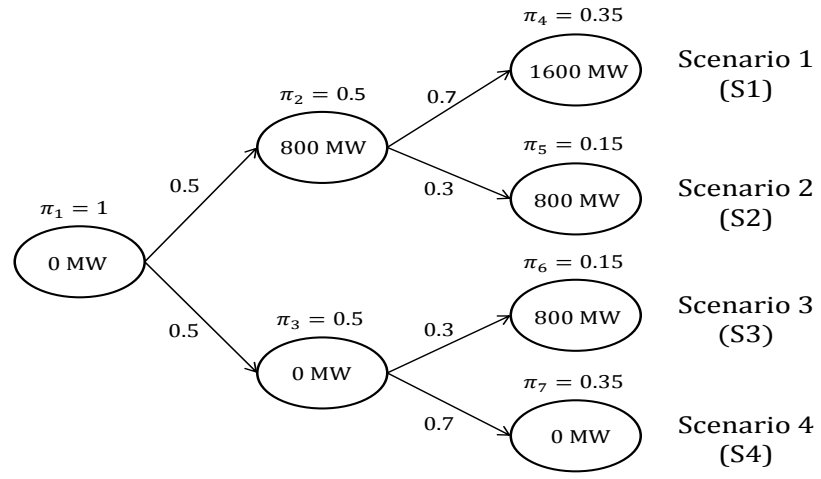

Fig. 1. Scenario tree for IEEE-RTS case study. Transition and node probabilities are shown.

In terms of investment, the planner can choose to invest in the conventional reinforcement options shown in Table I and alternative asset types shown in Table II. Conventional assets have been modeled with a build time delay of 1 epoch. Although they constitute an inexpensive way to accommodate oncoming generation, they suffer from 'information lag' and entail stranding risks. Note that each corridor can be upgraded only once per scenario. In contrast, storage devices and phaseshifters are assumed to be free of lengthy permission processes and can be deployed with minimum delay. Maximum angle for phase-shifters $\bar{\xi}$ is set to $30^{\circ}$, while storage devices have a total capacity $\bar{\eta}$ of $1600 \mathrm{MWh}$ and a maximum charge/discharge rate $\bar{h}$ of $400 \mathrm{MW}$. A penalty value $\Gamma$ of $30,000 £ / \mathrm{MWh}$ was used for all simulations. The choice of $\Gamma$ does not influence the simulation result as long as it is a large and positive value that ensures satisfaction of the intact and 
post-fault system balance equations without demand curtailment. In addition, a discount rate of 5\% has been used throughout; for example, the total cost for building reinforcement A over the 15 -year horizon is $£ 16.3 \mathrm{~m}$.

TABLE I

TRANSMISSION LINE REINFORCEMENT OPTIONS

\begin{tabular}{|c|c|c|c|}
\hline \multicolumn{4}{|c|}{ TRANSMISSION LINE REINFORCEMENT OPTIONS } \\
\hline \hline Asset Type & $\begin{array}{c}\text { Reinforcement } \\
\text { Capacity [MW] }\end{array}$ & $\begin{array}{c}\text { Annualized Capital } \\
\text { Cost [£/year] }\end{array}$ & Build Time \\
\hline \hline Option A & 200 & $1,500,000$ & 1 epoch \\
Option B & 400 & $2,500,000$ & 1 epoch \\
\hline \hline
\end{tabular}

TABLE II

ALTERNATIVE INVESTMENT OPTIONS

\begin{tabular}{|c|c|c|}
\hline \hline Asset Type & $\begin{array}{c}\text { Annualized Capital } \\
\text { Cost [£/year] }\end{array}$ & Build Time \\
\hline \hline Phase-shifter & 600,000 & 0 epochs \\
Storage device & $15,000,000$ & 0 epochs \\
\hline
\end{tabular}

In order to investigate system evolution under the deterministic and stochastic paradigms as well as quantify the benefit of considering investment in alternative asset types, we test three different models:

- D-I: Deterministic planning model where all asset types are allowed.

- S-I: Stochastic planning model where only investment in line reinforcements is allowed.

- S-II: Stochastic planning model where investment in all asset types is allowed.

Note that the stochastic model presented in Section III can be transformed to a deterministic model in a straightforward manner, by employing a scenario tree of 3 nodes and setting all node probabilities to 1 . All models have been developed using FICO Xpress 7.1 and run on a Xeon $3.46 \mathrm{GHz}$ computer, allowing for a parallel implementation on 10 processors. The Benders convergence criterion $\bar{\zeta}$ was set to $0.1 \%$. Table III displays investment decisions for all runs; for example 'A (39)' signifies reinforcement of the corridor connecting buses 3 and 9 with option A. Phase shifters are denoted PS and storage devices as STOR. In addition, we show investment (IC), operation (OC) and total costs (TC) for all runs. E\{ \} denotes the expectation operator.

\section{B. Optimal Deterministic and Stochastic Planning}

The deterministic case studies enable us to identify the optimal decisions when the planner has full information on future generation additions. Under the high-deployment scenario S1, the planner chooses to invest in the main wind exporting corridors (3-9), (3-24) and (15-24) from the very first epoch; the cost of first-stage commitments is $£ 70.8 \mathrm{~m}$. Some further investment take place in subsequent stages, taking advantage of phase shifters' capability for corrective control. Under S2 the planner invests again in the main exporting corridors, but this time option A is sufficient to accommodate the arising flows. The same conventional upgrades are chosen under S3, but the later wind deployment allows the planner to defer commitments to epoch 2. No investment is warranted under S4.

In contrast, case studies S-I and S-II employ the full stochastic formulation outlined in Section III. In the case of S-
I, the only first-stage commitment is to corridor (3-24) that connects the wind generator to high-load bus 3 . The decisions following the high-growth transition (from node $1 \rightarrow$ node 2 ) is a combination of option $\mathrm{A}$ and $\mathrm{B}$ reinforcements targeted at the same exporting corridors as in D-I. Additional investment in branches (14-16) and (15-16) are due to the lack of corrective control provision. Under S3 and S4, no further investments are made in later epochs.

When investment in alternative asset types is allowed, firststage commitments are deferred to the second epoch. In case of the high-growth transition, the wind-exporting bus 24 is equipped with a storage device that charges during high-wind periods and discharges in periods of high demand, considerably improving utilization of the available wind power. In addition, a mix of conventional reinforcements are built and commissioned in epoch 3 . Phase-shifters are also installed under both $\mathrm{S} 1$ and $\mathrm{S} 2$ to provide corrective control. In essence, the possibility for contingent deployment of flexible assets enables the planner to manage congestion due to the $800 \mathrm{MW}$ wind generation addition in node 2 without having to pre-maturely commit to a large investment in (3-24) as in S-I. The low-growth transition (node $1 \rightarrow$ node 3 ) does not warrant investment in storage. An investment in corridor (3-24) is preferred instead, but this time option $\mathrm{A}$ is chosen.

It is important to note that first-stage investment levels in both S-I and S-II are considerably lower when compared to the deterministic cases. This is because reinforcement decisions, especially when taken in the root node, entail a large risk of asset stranding; the planner chooses to be more conservative when facing uncertainty. This is relaxed in subsequent stages due to the inter-temporal uncertainty resolution. It is also important to underline that even though expected investment cost is higher in S-II, flexible assets lead to a reduction of total costs under all realizations. Naturally, expected total cost is lower in S-II and the option value of incorporating flexible assets is $£ 17.6 \mathrm{~m}$.

Another value of interest is the benefit of adopting the optimal stochastic strategy instead of naively following a deterministic plan when facing uncertainty. For the purposes of this comparison, we enforce first-stage decisions suggested by the four plans under D-I to the full stochastic model S-II and examine the extent to which the initial deterministic commitments can impede system development. Regarding the first-stage commitments suggested by D-I under S3 and S4, both studies recommend making no first-stage decisions, as also found in the optimal stochastic strategy. As a result, adopting one of these plans to guide first-stage decisions would not impose further costs. However, it is important to stress that this is essentially a coincidence and not a feature of deterministic planning. On the contrary, naively planning against a single eventuality can lead to over-commitment due to the inability to consider a 'wait-and-see' approach, resulting in substantial regret in the case of adverse realizations. This is the case when following the first-stage commitments suggested by $\mathrm{S} 1$ and $\mathrm{S} 2$, which entail considerable first-stage investments in conventional assets. 
TABLE III

Case Study InVestment Decisions And System Costs

\begin{tabular}{|c|c|c|c|c|c|c|c|c|c|c|}
\hline & \multicolumn{3}{|c|}{ Investment Decisions } & \multicolumn{6}{|c|}{ Costs (£m) } \\
\hline & & Epoch 1 & Epoch2 & Epoch 3 & IC & OC & TC & $E\{I C\}$ & $\mathbf{E}\{\mathrm{OC}\}$ & $\mathrm{E}\{\mathrm{TC}\}$ \\
\hline \multirow{4}{*}{$\ddot{n}$} & S1 & $\begin{array}{c}\text { A (3-9), B (3-24), } \\
\text { B (15-24) }\end{array}$ & $\begin{array}{c}\text { A (3-9), } \\
\text { PS (3-9), PS }(11-14)\end{array}$ & PS (15-16) & 91.3 & 4957.4 & 5048.8 & \multirow{4}{*}{44.9} & \multirow{4}{*}{5603.8} & \multirow{4}{*}{5648.7} \\
\hline & S2 & A (3-9), A (3-24), & PS $(11-14)$ & - & 52.9 & 5267.7 & 5320.6 & & & \\
\hline & S3 & - & $\begin{array}{c}\mathrm{A}(3-9), \mathrm{A}(3-24), \\
\mathrm{A}(15-24)\end{array}$ & $\begin{array}{c}\text { PS (9-12), PS (10-12), } \\
\text { PS }(11-13)\end{array}$ & 33.6 & 5834.9 & 5868.6 & & & \\
\hline & S4 & - & - & - & 0.0 & 6295.1 & 6295.1 & & & \\
\hline \multirow{4}{*}{ is } & S1 & $\mathrm{B}(3-24)$ & $\begin{array}{c}\mathrm{A}(1-3), \mathrm{A}(3-9), \mathrm{A}(14-16) \\
\mathrm{B}(15-16), \mathrm{B}(15-24)\end{array}$ & - & 87.6 & 5078.7 & 5166.3 & \multirow{4}{*}{57.4} & \multirow{4}{*}{5665.9} & \multirow{4}{*}{5723.3} \\
\hline & S2 & B $(3-24)$ & $\begin{array}{c}\mathrm{A}(1-3), \mathrm{A}(3-9), \mathrm{A}(14-16) \\
\mathrm{B}(15-16), \mathrm{B}(15-24)\end{array}$ & - & 87.6 & 5336.5 & 5424.1 & & & \\
\hline & S3 & B $(3-24)$ & - & - & 27.2 & 5897.1 & 5924.4 & & & \\
\hline & S4 & $\mathrm{B}(3-24)$ & - & - & 27.2 & 6295.1 & 6322.3 & & & \\
\hline \multirow{4}{*}{$\begin{array}{l}1 \\
\dot{1}\end{array}$} & S1 & - & $\begin{array}{c}\text { A (3-9), B (3-24), B (15-24), } \\
\text { PS (12-13), PS (16-19), } \\
\text { STOR (24) }\end{array}$ & $\begin{array}{c}\text { PS (3-9), PS (8-9), } \\
\text { PS (16-17) }\end{array}$ & 149.2 & 5009.9 & 5159.1 & \multirow{4}{*}{79.6} & \multirow{4}{*}{5626.1} & \multirow{4}{*}{5705.7} \\
\hline & S2 & - & $\begin{array}{c}\text { A (3-9), B (3-24), B (15-24), } \\
\text { PS (12-13), PS (16-19), } \\
\text { STOR (24) }\end{array}$ & PS (9-11), PS (10-12) & 147.6 & 5253.7 & 5401.3 & & & \\
\hline & S3 & - & $\mathrm{A}(3-24)$ & PS (9-11), PS (13-23) & 12.9 & 5875.4 & 5888.3 & & & \\
\hline & S4 & - & A $(3-24)$ & - & 9.5 & 6295.1 & 6304.6 & & & \\
\hline
\end{tabular}

By carrying out two further studies, we find that the corresponding expected system cost is $£ 5719.7 \mathrm{~m}$ and $£ 5708.9 \mathrm{~m}$ respectively. As a result, we can quantify the expected benefit of adopting a stochastic over a deterministic decision framework to determine first-stage commitments at $£ 5.4 \mathrm{~m}$. This further underlines the importance of incorporating strategic flexibility in early commitments through the use of a stochastic planning framework.

Finally, it is imperative to highlight that the benefits of enabling investment in flexible assets persist even when security constraints are not enforced. When ignoring N-1 constrains, system costs under S-I and S-II are $£ 5609.7 \mathrm{~m}$ and $£ 5596.6 \mathrm{~m}$ respectively, giving rise to a substantial option value of $£ 13.1 \mathrm{~m}$. Most notably, the main investment decision under S-II is the construction of a storage device at bus 24, similar to the strategy shown in Table III. This showcases that although corrective control provision is a significant advantage of the alternative technologies examined, the operational benefits stemming from the ability to control flows and manage the inter-temporal system-balance during intact operation can also be significantly large, warranting investment in their own right.

In its exhaustive formulation (i.e. no contingency screening), S-II is a MILP with 987 binary investment variables and 24.6 million continuous variables. In terms of model performance, S-II was solved in 30 minutes, after 4 iterations of the contingency-screening module. As discussed in Section III, contingencies were initially ignored and then binding operating points were progressively added. The first screening detected 17,840 binding points (out of a total possible of 229,320), with small further additions in the subsequent iterations. In an effort to solve the same problem with an exhaustive consideration of post-fault constraints, solution times exceeded the maximum time of 6 hours. This highlights the computational benefits of the proposed decomposition scheme for modelling N-1 secure operation.

\section{Discussion}

The presented case study has shown that deterministic plans that ignore uncertainty lead to long-term commitments so as to take full advantage of the scale economies present in transmission investment. Stochastic planning takes a more conservative approach, where first-stage commitments are reduced to minimize the cost of stranded assets. What is interesting to underline is that even though some particular investments (in this case storage) may be sub-optimal under deterministic studies, the introduction of uncertainty can render them valuable strategic options. Investment in flexible assets such as phase-shifters and storage devices with reduced build times render the planner capable of taking a 'wait-andsee' approach. Long-term commitments to line reinforcements can be deferred to the future, while interim system operation is facilitated and secured through the contingent deployment of flexible assets providing congestion management and corrective control.

\section{CONCLUSIONS AND FUtURE WORK}

The present paper describes a tool for transmission planning under generation uncertainty. We have extended the existing models (such as [13]) by considering a multi-stage scenario tree, investment in non-conventional assets that entail inter-temporal constraints, inclusion of $\mathrm{N}-1$ security constraints and by proposing a suitable decomposition scheme to render the problem tractable. It is important to note that even though the proposed modeling framework focuses on generation uncertainty, uncertainty in other system parameters, such as load growth or fuel prices can be accommodated in a straightforward manner.

Through a case study on IEEE-RTS we investigate the potential for non-conventional assets to accommodate new sources of renewable energy, analyze their benefits and demonstrate that such valuable investment opportunities can remain undetected under deterministic approaches. The 
envisaged low carbon future can be greatly facilitated by these technologies, granting planners the ability to react swiftly to the unfolding uncertainty and accommodate new generation in a timely manner, while limiting the need for anticipatory commitments.

In future work, there may be significant value in moving beyond the current risk-neutral formulation towards incorporating risk-averse decision criteria such as the minimization of the maximum regret experienced over all scenario realizations, in line with propositions in [15] and [29]. Another area for improvement is the consideration of uncertainty at operating timescales related to intermittent generation. Finally, an aspect of high interest that could be modeled is the fact that capital cost of new technologies tends to reduce not only over time, but may also depend on deployment rate. Such a study would underline the importance of exploring and analyzing all possible paths to a low-carbon future.

\section{REFERENCES}

[1] Council of the European Union, Energy and Climate Change - Elements of the final compromise, Dec. 2008.

[2] V. Rious, Y. Perez and J. Glachant, "Power transmission network investment as an anticipation problem", Review of Network Economics, vol. 10, no. 4, pp. 1-21, 2011.

[3] A.D. Lamont, "Assessing the economic value and optimal structure of large-scale electricity storage", IEEE Transactions on Power Systems, vol. 28, no. 2, pp. 911-921, May 2013.

[4] J. Mutale, G. Strbac, "Transmission network reinforcement versus FACTS: an economic assessment", IEEE Transactions on Power Systems, vol. 15, no. 3, pp. 961-967, August 2000.

[5] G. Strbac, "Demand side management: Benefits and challenges", Energy Policy, vol. 36, no. 12, pp. 4419-4426, 2008.

[6] E.E. Sauma and S.S. Oren, "Proactive planning and valuation of transmission investments in restructured electricity markets", Journal of Regulatory Economics, vol. 30, no.3, pp. 261-290, Nov. 2006.

[7] J. H. Zhao, Z.Y. Dong, P. Lindsay, K.P. Wong, "Flexible Transmission Expansion Planning With Uncertainties in an Electricity Market", IEEE Transactions on Power Systems, vol. 24, no. 1, pp. 479-488, Feb. 2009.

[8] L.G. Epstein, "Decision making and the temporal resolution of uncertainty", International Economic Review, vol. 21, no. 2, pp. 269283, Jun 1980.

[9] A.K. Dixit and R.S. Pindyck, Investment under uncertainty, Princeton University Press, 1994.

[10] G. Blanco, F. Olsina, F. Garces and C. Rehtanz, " Real option valuation of FACTS investments based on the least square Monte Carlo Method ", IEEE Transactions on Power Systems, vol. 26, no. 3, pp. 1389-1398, Aug. 2011.

[11] S.W. Wallace, "Stochastic programming and the option of doing it differently", Annals of Operations Research, vol. 177, no.1, pp. 3-8, 2010.

[12] T. Akbari, A. Rahimikian, A. Kazemi, "A Multi-stage Stochastic Transmission Expansion Planning Method", Energy Conversion and Management, vol. 52, no. 8, pp. 2844-2853, 2011.

[13] F.D. Munoz, B.F. Hobbs, J.L. Ho, S. Kasina, "An EngineeringEconomic Approach to Transmission Planning under Market and Regulatory Uncertainties: WECC Case Study", IEEE Transactions on Power Systems, vol. 29, no. 1, pp. 307-317, Jan. 2014.

[14] N. Growe-Kuska, H. Heitsch and W. Romisch, "Scenario reduction and scenario tree construction for power management problems", Proceedings of IEEE PowerTech Conference, Bologna, vol. 3, 2003.

[15] B.G. Gorenstin, N.M. Campodonico, J.P. Costa, M.V.F. Pereira, "Power system expansion planning under uncertainty", IEEE Transactions on Power Systems, vol. 8, no. 1, pp. 129-136, Feb. 1993.

[16] S. Ahmed, A.J. King, G. Parija, "A multi-stage stochastic integer programming approach for expansion under uncertainty", Journal of Global Optimization, vol. 26, no. 1, pp. 3-24, Apr. 2003.
[17] D. Pozo, J. Contreras, E. Sauma, "If you build it, he will come: Anticipative power transmission planning”, Energy Economics, vol. 36, pp. 135-146, 2013.

[18] D. Pozo, E. Sauma, J. Contreras, "A three-level static MILP model for generation and transmission expansion planning", IEEE Transactions on Power Systems, vol. 28, no. 1, pp. 202-210, Feb. 2013.

[19] A.J. Conejo, M. Carrión and J.M Morales, Decision Making Under Uncertainty, Springer, pp. 27-62, 2010.

[20] J.F Benders, "Partitioning procedures for solving mixed-variables programming problems", Numerical Mathematics, vol. 4, pp. 238-252, 1962.

[21] M. Shahidehpour, F. Yong, "Benders decomposition: applying Benders decomposition to power systems", IEEE Power and Energy Magazine, vol. 3, no. 2, pp. 20-21, Mar. 2005.

[22] F.D. Munoz, B.F. Hobbs and J.P. Watson, "New Bounding and Decomposition Approaches for Transmission and Generation Planning under Policy Constraints", John Hopkins University Working Paper. Available online at: https://jshare.johnshopkins.edu/bhobbs1/site/docs/papers/OR_Munoz_H obbs and Watson 2014.pdf

[23] J.R. Birge, F.V. Louveaux, "A multicut algorithm for two-stage stochastic linear programs", European Journal of Operational Research, vol. 34, no.3, pp. 384-392, 1988.

[24] F. Capitanescu, J.L. Martinez Ramos, P. Panciatici, D. Kirschen, A Marano Marcolini, L. Platbrood, L. Wehenkel, "State-of-the-art, challenges and future trends in security constrained optimal power flow", Electric Power Systems Research , vol. 81, no. 8, pp. 1731-1741, 2011.

[25] F. Bouffard, F.D. Galiana, J.M Arroyo, "Umbrella contingencies in security-constrained optimal power flow", 15th Power Systems Computation Conference (PSSC), Liege, vol. 5, 2005.

[26] F. Capitanescu, M. Glavic, D. Ernst, L.Wehenkel, "Contingency filtering techniques for preventive security-constrained optimal power flow", IEEE Transactions on Power Systems, vol. 22, no. 4, Nov. 2007.

[27] N. Alguacil, A.J. Conejo, "Multiperiod Optimal Power Flow using Benders Decomposition", IEEE Transactions on Power Systems, vol.15, no. 1, pp. 196-201, Feb. 2000

[28] Reliability Test System Task Force of the Application of Probability Methods Subcommittee, "IEEE reliability test system", IEEE Transactions on Power Systems, vol. 13, no. 6, pp. 2047-2054, Nov./Dec. 1979.

[29] A.H. van der Weidje and B.F. Hobbs, "The economics of planning electricity transmission to accommodate renewables: Using two-stage optimization to evaluate flexibility and the cost of disregarding uncertainty", Energy Economics, vol. 34, no.4, pp. 2089-2011, 2013.

\section{BIOGRAPHIES}

Ioannis Konstantelos (M'2012) received the MEng degree in Electrical and Electronic Engineering from Imperial College London in 2007. He obtained his $\mathrm{PhD}$ from the same university in 2013 in the field of electrical energy systems. His research interests include mathematical programming and statistical modeling techniques applied to planning of energy systems.

Goran Strbac is a Professor of electrical energy systems at Imperial College, London, UK. His current research interests include electricity generation, transmission and distribution operation, planning and pricing, and integration of renewable and distributed generation in electricity systems. 